\title{
Nanorobotics : a Newer Platform for Molecular Diagnose
}

\author{
Vijayakumar R, Jagannathan S, Rahul Gandhi P, Chaansha S \\ ${ }^{1 *}$ Department of Biochemistry, Karpagam Arts and Science College, Coimbatore, Tamilnadu. PIN 641021 INDIA. \\ ${ }^{2}$ Department of Tissue Culture Anti-Rabies Vaccine, Pasteur Institute of India, Coonoor, The Nilgiris, Tamilnadu. PIN 643103 INDIA. \\ ${ }^{3}$ Department of Biotechnology Jamal Mohammed College, Trichirapalli, Tamilnadu. PIN 621021 INDIA. \\ ${ }^{4}$ Department of Biotechnology, Kongunadu Arts and Science College, Coimbatore, Tamilnadu. PIN 641029 INDIA. \\ * Corresponding author: vijayakumar.thenilgiris@gmail.com(Vijayakumar R)
}

\section{Abstract}

The present era of nanotechnology has reached to a stage where scientist are able to develop and programme complex machines that are built at molecular level which can work inside the patient body. One such challenge is a nanorobot, which once thought to be a desire as came into reality now. The proposed application of nanorobot can range from common cold to dreadful diseases like cancer, Diabetes, influenza, cerebral aneurysm. This study of nanorobot serves as a lead to the field of nanomedicine. There are many applications for nanorobotic systems and its biggest impact would be in the area of medicine. This article deals with the nanorobotic design and their applications in molecular diagnosis.

Keywords: Nanotecehnology, Nanorobotics, Nanoscale, Nanomedcine, 3D, Signaling, Disease.

Citation: Vijayakumar R, et al. Nanorobotics: a Newer Platform for Molecular Diagnose. Nano Biomed. Eng. 2011, 3(3), 192-201. DOI: 10.5101/nbe.v3i3.p192-201.

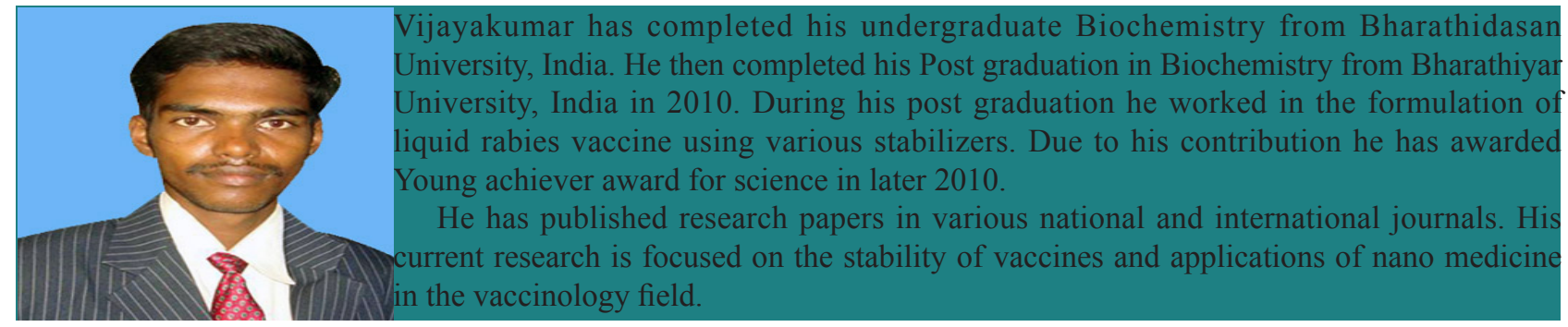

\section{Introduction}

In the recent trend, development of biotechnology is influential in the diagnosis of the threatening diseases, in deed to diagnose the threatening disease. According to the world health organization infectious disease accounts for approximately $25 \%$ of all deaths worldwide, $45 \%$ of death in low income countries and $63 \%$ of children worldwide within a short period. Worldwide infectious and microbial diseases account for approximately $40 \%$ of the total 50 million annual deaths. To decrease the mortality rate of threatening diseases by vaccination. Although vaccination delivered by injection as led to tremendous advances in the control of many infectious diseases. This technique as risk, discomfort. It escort to such for alternative source for vaccine delivery, which advances the immunobiologicals delivery newer needle free vaccines may decrease poor complaints with schedules. But the needle free injection not yet on the perspective but should be pursed. So that the safety needle based immunobiologicals systems can be maximized, and also it have some timeframes, with that timeframe each technology passes through the phases of research and development, product, launch, market development and post market monitoring [1].Traditional methods for clinical analysis of contamination is useful to positively identify the infected with some sort of virus, this laboratorial process demands a precious time and a complex infrastructure. The 'Nanos' meaning dwarf, is a prefix used to describe "one billionth" of something, or 0.000000001 . The term Nanotechnology was first defined by Tokyo Science University professor Norro Taniguchi in 1974. Nanotechnology is often represented by two fundamentally different approaches: top down and 
bottom up. Top down refers to make nanoscale structures by machining, templating and lithographic techniques and bottom up refers to build organic and inorganic materials into atom by atom and molecule by molecule [2]. Nanotechnology has emerged as a big potential technology for delivery of drugs at a specific site. Drugs, proteins, vaccines and nucleotides can be delivered more appropriately to attain desired pharmacological effects by Nanotechnology. Nanotech components form the base for drug delivery systems that will hopefully transport therapeutic or diagnostic agents to specific sites in the body allowing highly targeted treatments that could minimize the side effects. Nanotechnology is the next step after miniaturization.

\section{Applications of nanotechnology}

Nanotechnology has developed in the field of electronics, space, food, textile, optics and medicine. In the field of medicine, the technology has developed with various aspects such as drug delivery, tissue engineering and manufacturing of nanorobots for the diagnosis of various diseases. The biomarkers, micro arrays could actually be widely used for the diagnosis of diseases like cancer, diabetes, cardiac and brain disease. (Fig. 1) [3].

\subsection{Food}

Nanotechnology is having an impact on several aspects of food science, from how food is grown to how it is packaged. Companies are developing nonmaterial's that will make a difference not only in the taste of food, but also in food safety, and the health benefits that food delivers.

\subsection{Medicine}

Researchers are developing customized nanoparticles, the size of molecules that can deliver drugs directly to diseased cells in the body. When it's perfected, this method should greatly reduce the damage caused by the treatments such as chemotherapy does to a patient's healthy cells. Nanomedicine refers to future developments in medicine that will be based on the ability to build nanorobots. These nanorobots are programmed to repair specific diseased cells, functioning in a similar way to antibodies in our natural healing processes.

\subsection{Nanotechnology in drug delivery}

Nanotechnology provides a unique possibility to create systems that can better deliver drugs to tiny areas within the body. Nano enabled drug delivery also makes it possible for drugs to permeate through cell walls, which critical importance to the expected growth of genetic medicine over the next few years. Drug encapsulating nanoparticles offer extensive control over delivery location, drug dosage, drug release characteristics is possible. Bioadhesive and drug-free nanoparticles may be used to reduce infection in certain cases of diseases.
Beyond the obvious application of nanotechnology to medicine, the approach is fundamentally different than conventional medicine. Nanomedicine is the treatment or repair of tissues and organs, within individually targeted cells, cell-by-cell (a nano "bottoms up", rather than topdown approach). It is typically combines use of molecular biosensors to provide feedback control of treatment and repair. Most conventional medicine does not use feedback control. Drug use is targeted and adjusted appropriately for individual cell treatment at the proper dose for each cell.

\section{5 impact of Nanomedicines}

Impact of nanomedicine in the long-term, end of pain, end of physical disease and restoration of "youthful" health to aged. As usual there's an equal downside: technology is usable in nanobiowarfare. Cost of nanomedical care similar to other "luxury" goods. Now: infinite early on: extremely high over time: decreasing eventually: very low some experts believe this costreduction process could happen quickly [4].

\section{Nanorobotics}

A new approach within advanced graphics simulations is presented for the problem of nano-assembly automation and its application for medicine. The problem under study concentrates its main focus on nanorobot control design for molecular manipulation and the use of evolutionary agents as a suitable way to enable the robustness on the proposed model. Biological-based robots can be diverted by microbes that can piggyback on its metabolism, and can even cause the engineered biomachine to perform some new or different function than was originally intended. Biological based robots may be found to be unpatentable for the pure fact that they are biologically based. Mechanical robots will have no such difficulty in receiving patents. Its provides useful directions for further research and development of medical nanorobotics and suggests a time frame in which nanorobots may be expected to be available for common utilization in therapeutic and medical procedures (Fig. 2) [5].

\subsection{Distinctive properties of Nanorobotics}

Nanosclae materials used to diagnose the disease in rapid and sensitively. The nanoparticles also involve in the diagnosis of action of diseased cells. The various chemical reactivity of nanoscale materials greatly different from more macroscopic form, e.g., gold. Vastly increased surface area per unit mass, e.g., upwards of $100 \mathrm{~m}^{2}$ per gram. Quantum size effects result in unique mechanical, electronic, photonic, and magnetic properties of nanoscale materials. New chemical forms of common chemical elements, e.g., fullerenes, nanotubes of carbon, titanium oxide, zinc oxide, and other layered compounds.

\subsection{Dendrimers}

\subsection{Nanomedicines}




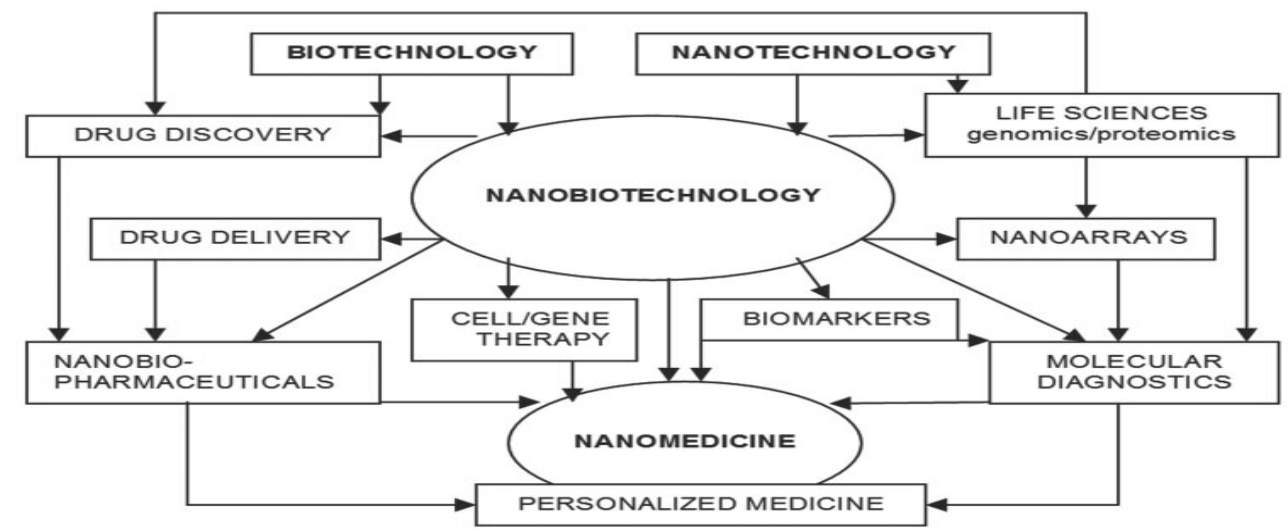

Fig. 1 Applications of nanotechnology

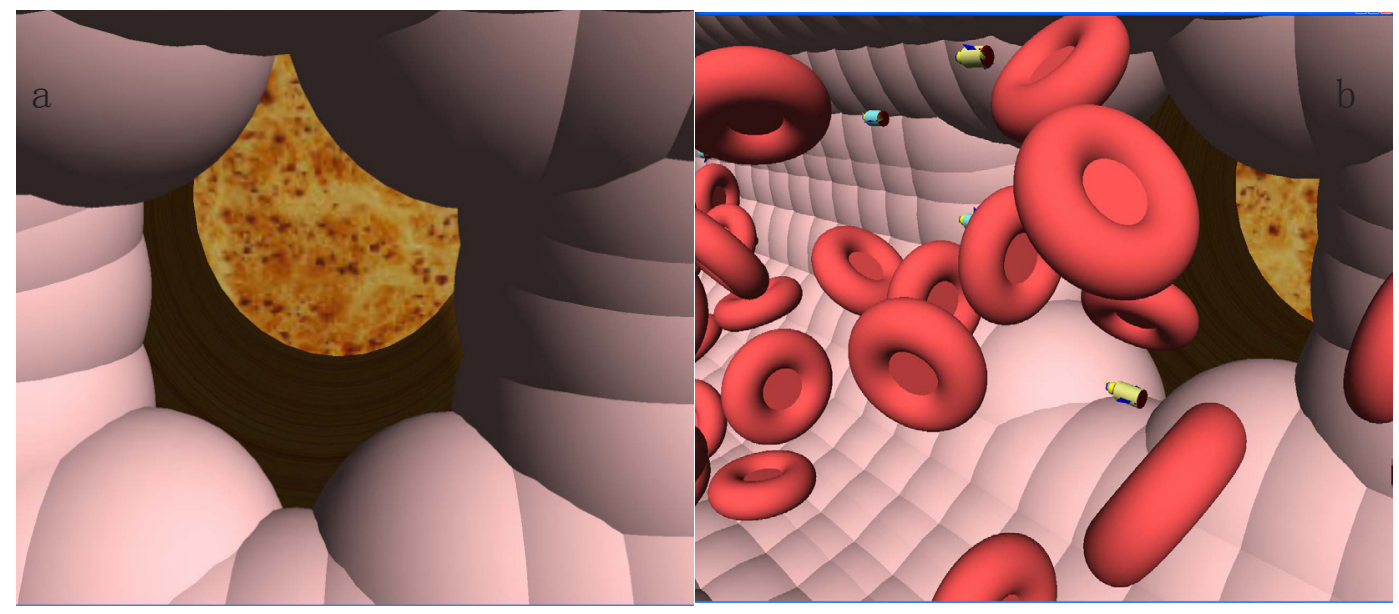

Fig. 2 (a) Aneurysm at the initial stages of development. (b) Nanorobots flowing near the aneurysm detects NOS signal [5]

The dendrimers are perfect and monodispersed macromolecules with regular highly branched 3D architecture. They offer controlled delivery and targeting. It possesses three components: Initiator core, interior layers and externally attached core. Eg: poly amido amino (PANAM) dendrimers, poly-propyleneimine, tecto dendrimers, multi-lingual dendrimers and chiral dendrimers. Dendrimers are used in microcapsules; nanodevice, and liposomes etc., nanodevices targeted into tumor cells through h-foliate receptor have been designed using dendrimers [2].

\subsection{Quantum dots}

Quantum dots are tiny crystals and they glow when stimulated by UV light. The wavelength or color of light depends on size of crystals. Latex beads filled with these nanoscale semiconductor dots can be design to level specific DNA sequence. The crystals are stimulated UV light each bead emits light that serves as a particular region of DNA [2].

\subsection{Cantievers}

Nanoscale cantilevers microscopic flexible beams resembling a row of dividing boards, a built using lithographic techniques and are coated with molecules capable of binding biomarkers of cancer like PSMA (Prostate -Specific Membrane Antibody). As Cancer cell secrete its molecular products, the antibodies coated on the cantilever fingers selectively bind to these secreted proteins and signaling the presence of cancer [2]

\subsection{Nanoshells}

Nanoshells have a core of silica and metallic layer. These nanoshells can be linked to antibodies that can recognize tumor cells (PSMA). Once the cancer cells take them up, by applying a near infrared light that is absorbed by the nanoshells, it is possible to create intense heat and that selectively kills tumor cells and not the neighbouring healthy cells.[6].

\subsection{Design of nanorobotic systems}

For making of nanorobot it needs detail such as, proteins and DNAs. There are many complexities which are associated with using biocomponents (such as protein folding, presence of aqueous medium), but the advantages of using these are also quite considerable. These bio components offer immense variety and functionality at a scale where creating a man made material with such capabilities would be extremely difficult. These bio components have been perfected by nature through millions of years of evolution and hence these are very accurate and efficient. The other significant advantages in using protein-based bionano components is the 


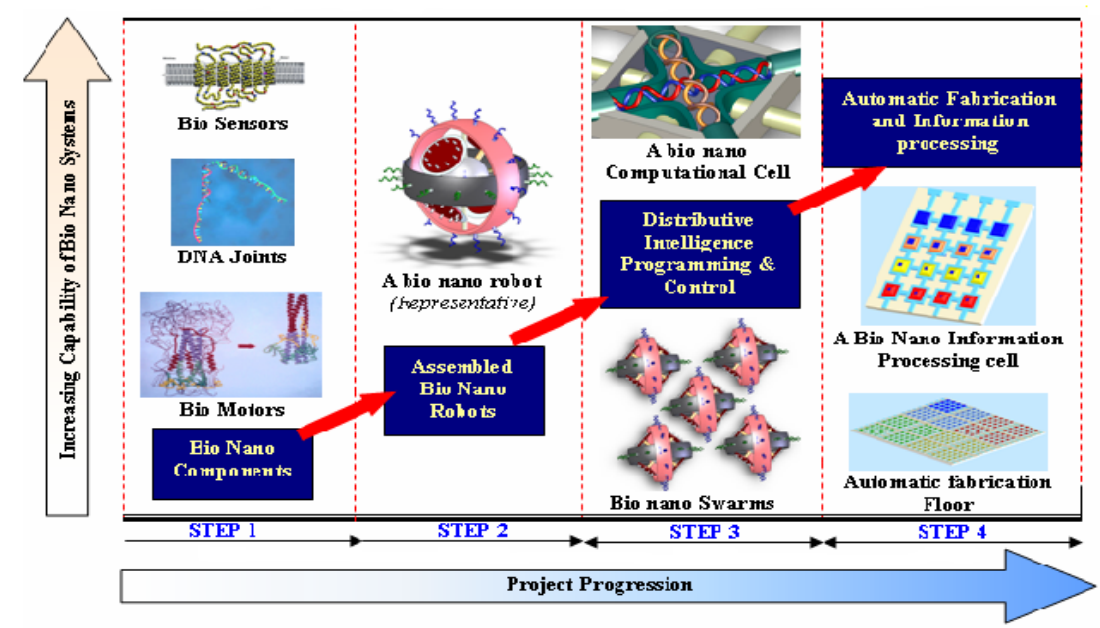

Fig. 3 The Roadmap, illustrating the system capability targeted as the project progresses [8].

development and refinement over the last 30 years of tools and techniques that enable researchers to mutate proteins in almost any way imaginable [7]. Hence, these biocomponents seem to be a very logical choice for designing nanorobotics. Some of the core applications of nanorobots seem to be a good choice as they both offer efficiency and variety of functionality. This idea is clearly inspired by nature's construction of nanorobots, bacteria and viruses which could be termed as intelligent organisms capable of movement, sensing and organized control.

\section{The roadmap of nanorobotics}

A roadmap is proposed which details the main steps towards the design and development of bio-nanorobots (Fig. 3). The roadmap progresses through the following steps.

\section{Step 1: Bio Nano Components}

From the simple elements such as structural links to more advanced concepts such as motors, each component must be carefully studied and possibly manipulated to understand the functional limits of each one of them. DNA and carbon nanotubes are being fabricated into various shapes, enabling possibilities of constructing newer and complex devices [8]. These nano-structures are potential candidates for integrating and housing the bionano components within them. Proteins such as rhodopsin and bacteriorhodopsin are a few examples of such bionano components.

\section{Step 2: Assembled Bionano Robots}

The next step involves the assembly of functionally stable bionano components into complex assemblies. A single bionanorobot will have actuation, sensory and information processing capabilities. The modular organization defines the hierarchy rules and spatial arrangements of various modules of the bionanorobot such as: the inner core (energy source for the robot); the actuation unit; the sensory unit; and the signaling and information-processing unit. By the beginning of this phase a "library of bionano components" will be developed, which will include various categories such as, actuation, energy source, sensory, signaling etc [8]. There will be systems that will sense an oxygen deprivation and stimulate other components to generate oxygen, creating an environment with stable homoeostasis.

\section{Step 3: Distributive Intelligence, Programming and Control}

With the individual bionanorobot in full function, they will now need to collaborate with one another to further develop systems and "colonies" of similar and diverse nanorobots. This design step will lay the foundation to the concept of bio-nano swarms (distributive bionanorobotics).Designing swarms of bionanorobot capable of carrying out complex tasks and capable of computing and collaborating amongst the group will be the focus [8]. This basic computational cell will initially be designed for data retrieval and storage at the nano scale. This capability will enable us to program the swarm behavior in the bionano robots. This programming capability would form the core essence of a bio-nano robotics system and hence enables them with immense power.

\section{Step 4: Automatic Fabrication and Information Processing Machines}

For carrying out complex missions, such as sensing, signaling and storing, colonies of these bio-nanorobotic swarms needs to be created. The next step in nanorobotic designing would see the emergence of automatic fabrication methodologies of such bionano robots in vivo and in vitro. Capability of information processing, which will involve learning and decision making abilities, will be a key consideration of this step. These swarms could be programmed to search for alternate energy sources and would have an ability to adapt as per that resource. Energy management, self-repairing, and evolving will be some of the characteristics of these swarms [8].

\subsection{Testing and diagnosis}


Nanorobotics will make the results of diagnosis available in a matter of seconds with a much wider range of testing abilities. For instance, a device the size of a calculator with a probe with billions of nanoscale molecular receptors mounted on hundreds of selfguiding retractile stalks will be able to be inserted in to the human body using nanomedicine. Comprehensive genotyping and real-time whole-body scans for particular bacteria, tumor cell antigens, mineral deposits, suspected toxins, hormone imbalances of genetic or lifestyle origin, and other specified molecules, producing 3D maps of desired targets of patients can be obtained. Up till now, our medical intervention was mainly to assist the body in healing itself. Nanorobotics will gives us systemic multilevel access to our internal physical and mental states, including real-time operating parameters of our own organs, tissues and cells. There is also the capability of programming such things as sexual preference, shyness or boldness, criminal propensity, personality disorders, and phobias.

\subsection{Nanorobots manufacturing technology}

The ability to manufacture nanorobots can be understood as the result from current trends and new methodologies in fabrication, computation, transducers and manipulation. Depending on the case, different gradients on temperature, concentration of chemicals in the bloodstream, and electromagnetic signature are some of relevant parameters for biomedical purposes. The CMOS industry is successfully showing a pathway for the assembly processes needed to manufacture components required to enable nanorobots, where the joint use of nanophotonic and nanotubes is even accelerating further the actual levels of resolution ranging from $248 \mathrm{~nm}$ to $157 \mathrm{~nm}$ devices $[9,42]$.

\subsection{Chemical sensor}

Complimentary Metal Oxide Semiconductor (CMOS) integrated sensors using nanowires as material for circuit assembly can achieve maximal efficiency for applications regarding chemical changes, enabling new medical applications [10]. To further advance manufacturing techniques, Silicon-On- Insulator (SOI) technology has been used to assemble high performance logic sub $90 \mathrm{~nm}$ circuits. The protein Nitric Oxide Synthase (NOS) can provide positive or negative effects upon cell and tissues in their cellular living processes. It was also established the correlations between higher levels of NOS and brain aneurysm were established. Carbon nanotubes serve as ideal materials for the basis of CMOS integrated circuits (IC) based nanobiosensors. For a medical nanorobot, the use of CMOS as an actuator based on biological pattern is adopted in our architecture as a natural choice. Ion channels can interface electrochemical signals using sodium for the energy generation, which is necessary for mechanical actuators operation. Embedded actuators are programmed to perform different manipulations, enabling the nanorobot a direct active interaction with the bloodstream patterns and the molecular parameters inside the body. [11].

\subsection{Nano manipulation - virtual reality based design techniques}

The virtual reality is essential to visualize the atom-toatom interaction in real-time and see the results in a fully immersive 3D environment. Also, to facilitate user input in nanorobotic systems it is essential to develop voice, gesture and touch recognition features in addition to the conventional visualization and manipulation techniques. Virtual Reality (VR) technology comes to our aid by providing the experience of perception and interaction with the nano world through the use of sensors, effectors and interfaces in a simulated environment. These interfaces transform the signals occurring at nano-scale processes into signals at macro-level and vice-versa. The requirement is that the communication with the nanoworld must be at high-level and in real-time, preferably in a natural, possibly intuitive 'language'. Considering the nano-specific problems related to task application, tools and the interconnection technologies it leads to many flexible nano manipulation concepts [12].

Atomic Force Microscope (AFM) studies in biotechnology results from the fact that AFM techniques offer several unique advantages: first, they require little sample preparation, with native biomolecules usually being imaged directly; second, they can provide a $3 \mathrm{D}$ reconstruction of the sample surface in real space at ultra-high resolution; third, they are less destructive than other techniques and fourth, they can operate in several environments, including air, liquid, and vacuum. The operation of AFM in aqueous solution offers a unique opportunity for imaging biological molecules and cells in their physiological environments and their biologically important dynamic processes in real time [13]. Construction of a VR space in an off-line operation mode for trajectory planning combined with a real-time operation mode for vision tracking of environmental change ensures a complete 'immersed' visual display. Example of 3D nano manipulation system with a 3D VR model of the environment including the bio cell, and carrying out the user viewpoint change in the virtual space [14].

\section{Medical nanorobots}

The research and development of nanorobots with embedded nanobiosensors and actuators is considered a new possibility to provide new medical devices for doctors [15]. As integrated control mechanisms at microscopic environments differ from conventional control techniques, approaches using event-based feed forward control are sought to effectively advance new medical technologies. In the same way the development of bioelectronics in the 1980 s has led to new tools for biomedical instrumentation, the manufacturing of nanoelectronics [16]. The use of microdevices in surgery 
and medical treatments is a reality, which has brought many improvements in clinical procedures in recent years. A first series of nanotechnology prototypes for molecular machines are being investigated in different ways [17]. More complex molecular machines, or nanorobots, having embedded nanoscopic features represent new tools for medical procedures.

\subsection{Applications of medical nanorobots}

The use of nanorobots may advance biomedical intervention with minimally invasive surgeries [18], help patients who need constant body function monitoring, and improve treatment efficiency through early diagnosis of possibly serious diseases. Implantable devices in medicine have been used for continuous patient data acquisition. Patient monitoring can help in preparing for neurosurgery [19], early stage diagnostic reports to fight cancer, and blood pressure control for cardiology problems. The same approach is quite useful in monitoring patients with diabetes.

\subsubsection{DNA based nanorobots}

Nature choose DNA mainly as an information carrier, there was no mechanical work assigned to it. Energy conversion, trafficking, sensing, etc., there are certain qualities that make DNA an attractive choice for the construction of artificial nanomachines. In recent years, DNA has found use in not only mechanochemical, but also in nanoelectronic systems as well [20]. A DNA double-helical molecule is about $2 \mathrm{~nm}$ in diameter and has 3.4-3.6 nm helical pitches no matter what its base composition is; a structural uniformity is not achievable with protein structures if one changes their sequence. Furthermore, double-stranded DNA (ds-DNA) has a respectable persistence length of about $50 \mathrm{~nm} \mathrm{[37]} \mathrm{which}$ provides it enough rigidity to be a candidate component of molecular machinery. Single stranded DNA (ss-DNA) is very flexible and cannot be used where rigidity is required; however, this flexibility allows its application in machine components like hinges or nanoactuators. Its persistence length is about $1 \mathrm{~nm}$ covering up to three base pairs at $1 \mathrm{M}$ salt concentration.

Dr. Nadrian Seeman and colleagues built the first artificial DNA-based structure in the form of a cube in 1991. They then created more complex structures such as knots and Borromean rings [21]. In addition to these individual constructs, $2 \mathrm{D}$ arrays with the help of the double-crossover (DX) DNA molecule This DX molecule gave the structural rigidity required to create a dynamic molecular device, the $\mathrm{B}-\mathrm{Z}$ switch . The base $\mathrm{CG}$ repeated regions can be switched between the left and the righthanded conformations by changing ionic concentration [22]. The switch was designed in such a way that it had three cyclic strands of DNA, two of them wrapped around a central strand that had the CG-repeat region in the middle. The DNA based nanomachines and nanorobotics were used to identify the cancerous region of the body parts. These DNA based nanorobotics are the markers in the detection of many type of cancers.

\subsubsection{Applications of nanorobots in disease diagnosis}

Nanorobots are expected to enable new treatments for patients suffering from different diseases, and will result in a remarkable advance in the history of medicine. Studies targeted at building biosensors and nanokinetic devices [23], required to enable medical nanorobotics operation and locomotion, have also been progressing. The use of nanorobots may advance biomedical intervention with minimally invasive surgeries, and help patients who need constant body functions monitoring, or ever improve treatments efficiency through early diagnosis of possible serious diseases [24].

The nanorobots may be utilized to attach on transmigrating inflammatory cells or white blood cells, thus reaching inflamed tissues faster to assist in their healing process. Nanorobots will be applied in chemotherapy to combat cancer [25], through precise chemical dosage administration, and a similar approach could be taken to enable nanorobots to deliver anti-HIV drugs. Nanorobots could be used to process specific chemical reactions in the human body as ancillary devices for injured organs. Monitoring diabetes and controlling glucose levels for patients will be a possible application of nanorobots [26].

\subsubsection{Nanorobotics in disease}

Patients with diabetes must take small blood samples many times a day to control glucose levels. Such procedures are uncomfortable and extremely inconvenient. Serious problems may affect the blood vessels if the correct target levels of glucose in the blood are not controlled appropriately. Improper glucose control may result in a large range of consequences for the nervous system, kidney, and eyes, aggravate heart problems, and can even lead to stroke [25]. The level of sugar in the body can be observed via constant glucose monitoring using medical nanorobotics. This important data may help to supervise and improve the patient medication and diary diet. The glycemic levels and parameters for an adult with diabetes stay inside the desired ranges, the patients must try to keep their glucose level between 90-130 $\mathrm{mg} \mathrm{dL}^{-1}$ before refection, and $<180 \mathrm{mg} \mathrm{dL}^{-1}$ after refection. Upon waking the expected blood pressures should be $<130 / 80 \mathrm{mmHg}$. The glaciated hemoglobin (A1C) time series results must stay $<7.0 \%$. As a result of good blood sugar levels a red blood cell life span is 120 days. For diabetes application, the most important use of nanorobots is monitoring daily the patient's glucose levels, if possible without interfering in their way of life. Thus, one mobile phone is enough for data transferring and monitoring purposes. Whether the doctor wants to track the nanorobots current positions due to some medical reason, it can be done clinically or at home with at least two additional transmitters, which may comprise other ancillary preprogrammed cellular phones 

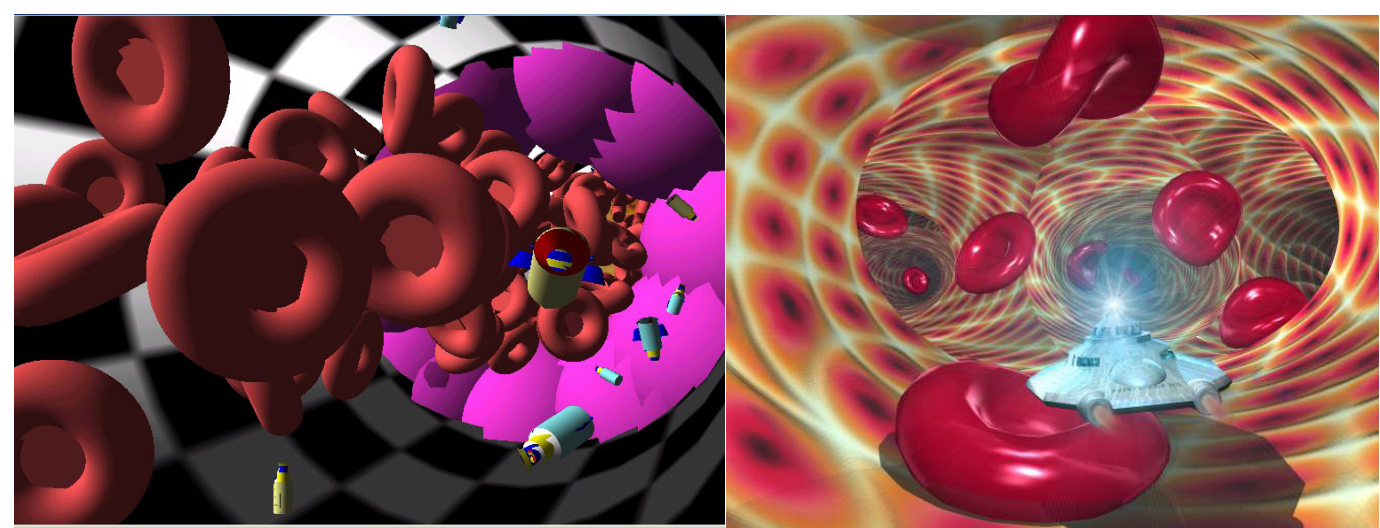

Fig. 4 (a): View of the NCD simulator workspace showing the inside view of the occluded LAD coronary artery, red blood cells and nanorobots. (b) Nanobots to Clean Arteries.[26]

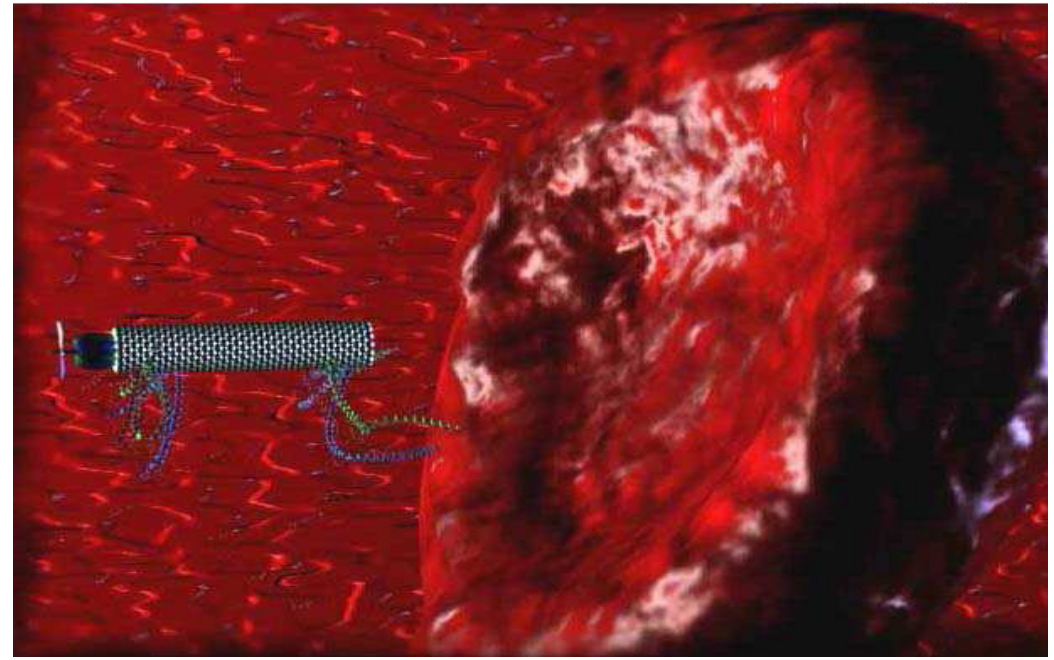

Fig. 5 Insect Nanorobots in treatment of cancer [36]

or transponder devices [28].

\subsubsection{Treatment of artery occlusion}

An important possible feature of medical nanorobots will be the capability to locate atherosclerotic lesions in stenosed blood vessels, particularly in the coronary circulation, and treat them mechanically, chemically or pharmacologically [29]. Cardiovascular problems are generally correlated with the obesity, human sedentary lifestyle, or hereditary characteristics. Heart problem is the world biggest killer. The bloodstream keeps the human body alive. In the blood, suspended in the plasma, is found the white blood cells, red blood cells, and platelets. The plasma represents $55 \%$ of the blood volume which is $8 \%$ of the body weight, with the size of red blood cells is about $7.5 \mu \mathrm{m}$ in diameter and $2 \mu \mathrm{m}$ thick. Platelets are 2 to $4 \mu \mathrm{m}$ in diameter [30]. The heart keeps the closed circulatory system working continuously. The bloodstream flows with the pumping system comprised of a closed system of blood vessels. The heart performs a key role for the human wellbeing, delivering $\mathrm{O}_{2}$ to large range of tissues, which returns $\mathrm{CO}_{2}$ to the lungs. The blood is pumped from the left ventricle through the arteries and arterioles to the capillaries. After that, the blood flows from venules into the veins back to the right atrium completing the systemic circulation. In the right atrium the blood is pumped through the lungs. The lungs equilibrate the $\mathrm{O}_{2}$ and $\mathrm{CO}_{2}$ in the alveolar air. The sounds produced by the heart are the result of diastolic and systolic pressures, which comes from the partially on striated vibrating arteries motions. The fluid in the Left Anterior Descending (LAD) moves with velocity $~ 38$ $\mathrm{cm} \mathrm{s}^{-1}$ (Fig. 4), as is typical of flow in aorta and arteries vessels [31].

The nanorobots must be equipped with the necessary devices for monitoring the most important aspects of disease pathogenesis. Depending on the case different gradients on temperature, concentration of chemicals in the blood stream and electro magnetic signature are some of the relevant parameters when monitoring patients. Teams of nanorobots may cooperate to perform computing processing, energy supply and data transmission capabilities can be addressed through embedded integrated circuits, using advances on technologies derived from very large scale integration (VLSI) design [32].

\subsubsection{Influenza characteristics}


For influenza, the contamination can happens through inhalation, ingestion, or direct contact through hand shaking and conversation. The influenza virus invades cell, and after the cell invasion, it makes use of enzymes to decrease intracellular $\mathrm{pH}$, slightly increasing $\sim 10 \mathrm{C}$ intracellular temperature, which is used to accelerate virus cell fusion activity [33]. Before a person shows symptomatic reactions, short after being infected by influenza, the bloodstream begins to receive a higher concentration of alpha- $\mathrm{N}$-acetylgalactosaminidase (alphaNAGA), which is secreted from the invaded cells. The protein hemagglutinin serves as virus envelope for influenza, promoting alpha-NAGA signals. Alpha-NAGA is a protein identified through the genome mapping, which belongs to chromosome 22. The lack of macrophage, incurred from the alpha-NAGA enzyme secreted through the infected cells, leads to immunosuppression and helps the virus to spread easily through the body. Thus, this change of chemical concentration, with over expression of alpha-NAGA in the bloodstream, is used to trigger the nanorobot prognostic behavior, which sends electromagnetic back propagated signals to the mobile phone carried with the person. As an integrated biohazard defense system, once the nanorobot activated the cell phone, this information is retransmitted for the satellites utilized as feasible telecommunication system [34].

\subsubsection{Nanorobots in cancer detection and treatment}

Nanorobots could be a very helpful and hope full and therapy of cancer patients, since current treatments like radiation therapy and chemotherapy often end up destroying more healthy cells than cancerous ones. From this point of view, it provides a non- depressed therapy for cancer patients. The nanorobots will be able to distinguish between different cell types that is the malignant and the normal cells by checking their surface antigens. This is accomplished by the use of chemotactic sensors keyed to a specific antigen on the target cells (Fig 5). Using chemical sensors they can be programmed to detect different levels of E- Catherin and beta-catenin in primary and metastatic phases. Medical nanorobots will then destroy these cells, and only these cells. The following methods were considered.

Random: nanorobots moving passively with the fluid reaching the target only if they bump into it due to Brownian motion.

Follow gradient: nanorobots monitor concentration intensity for E- cadherin signals, when detected, measure and follow the gradient until reaching the target. If the gradient estimate subsequent to signal detection finds no additional signal in $50 \mathrm{Ms}$, the nanorobot consider the signal to be a false positive and continuous flowing with the fluid.

Follow gradient with attractant: as above, but nanorobots arriving at the target, they release in addition a different chemical signal used by others to improve their ability to find the target. Thus, a higher gradient of signal intensity of E-cadherin is used as chemical parameter identification in guiding nanorobots to identify malignant tissues. Integrated nanosensors can be utilized for such a task in order to find intensity of E-cadherin signals. Thus they can be employed effectively for treating cancer [35].

\subsubsection{Nanorobotics control in brain aneury-}

\section{sms}

Endovascular treatment of brain aneurysms, arteriovenous malformations, and arteriovenous fistulas are biomedical problems expected to benefit from current research and developments in the field medical nanorobotics. The advent of biomolecular science and new manufacturing techniques is advancing the miniaturization of devices from micro to nanobioelectronics. A first series of nanotechnology prototypes for molecular machines are being investigated in different ways in the diagnosis of brain aneurysm. More complex molecular machines, or nanorobots, having embedded nanoscopic features represent new tools for medical procedures [36] Sensors for biomedical applications are advancing through teleoperated surgery and pervasive medicine, and this same technology provides the basis for manufacturing biomolecular actuators. The parameters used for the medical nanorobot architecture prototyping and its control activation, as well as the required technologies that address the manufacturing hardware background for molecular machines in brain aneurysm [26]. For analysis, a real time simulation based on clinical data is implemented, demonstrating sensor and nanorobot behavior capabilities for detection of abnormal vessel dilatation in cases of cerebral aneurysm. The use of real time 3D prototyping and simulation are important tools for medical nanorobots research and development. Such tools have significantly helped the semiconductor industry to achieve faster VLSI development [37].

\section{Control of nanorobotics systems}

The control of nano robotic systems could be classified in two categories:

i. Internal control mechanisms

ii. External control mechanisms

The other category could be the hybrid of internal and external control mechanisms.

\section{Internal Control Mechanism - Active and Passive}

This type of control depends upon the mechanism of biochemical sensing and selective binding of various biomolecules with various other elements. This is a traditional method, which has been in use since quite sometime for designing biomolecules. Using the properties of the various biomolecules and combining with the knowledge of the target molecule that is to be influenced, these mechanisms could be effective. But again, this is a passive control mechanism where at run 
technologies are enabling innovative works and patents which may help in constructing and employing nanorobots most effectively for biomedical problems. The implemented 3D simulator is a practical tool for exploring new techniques, nanomanufacturing strategies and nanorobot mobility considerations including actuation and data transmission, helping designers to define the appropriate molecular machine architecture. The joint use of nanophotonic and nanotube-based technologies may further accelerate the actual levels of CMOS resolution ranging down to $45 \mathrm{~nm}$ devices.

The future of bio nanorobots (molecular robots) is bright. We are at the dawn of a new era in which many disciplines will merge including robotics, mechanical, chemical and biomedical engineering, chemistry, biology, physics and mathematics so that fully functional systems will be developed [8].

Over the next five to ten years' biotechnology will make possible even more remarkable advances in molecular medicine and biobotics-microbiological robots/ engineered organisms [41].The first half of the $21 \mathrm{st}$ century nanomedicine will eliminate all common diseases of the 20th century. In the 3D stimulation, the nanorobots were able to detect efficiently alpha-NAGA signals in the blood stream with the integrated system retrieving information from a person infected with influenza. 3D stimulation provides design for manufacturability, major control interface requirements, and inside body molecular sensing for practical development and application of nanorobots in medical prognosis, which is an effective way to avoid an aggressive pandemic disease to spread. The research and development of nanorobots for common application in fields such as medicine and defense technology should lead as for a safer and healthier future [11], nanorobots are also candidates for industrial applications. The advent of molecular nanotechnology will again expand and enormously the effectiveness, comfort and speed of future medical treatments while at the same time significantly reducing risk, cost and invasiveness [35]. The higher dedications for the development of nanorobotics will leads to improvisation of personalized medicines within the time frame.

\section{References}

1. Jagannathan S,Chaansha S, Rajesh K, Santhiya T, Jayaraj D, Iyappan S R. A novel approaches in delivering immunobiologicals: A Glimpse. Advanced Biotech. 2009; 8(11):22- 31.

2. Parakh S R, C Swati C Jagdale, S Namita S Dodwadkar, Kashyap D Savalia. . Nanotechnology. The Indian pharmacist. 2008; 15-18.

3. Kewal K Jain. .Applications of Nanobiotechnology in Clinical Diagnostics. Clinical Chemistry, Oak ridge conference. 2007; 53(11):2002-2009.

4. Christine Peterson. Nanomedicine: the most challenging application of advanced nanotechnology, The Frontier of the Invisible. 2004; 21-29.

5. Adrino Cavalcanti et al. 2007 IEEE International conference on Nanotchnology Hardware Architecture for Nanorobot Application in Cerebral Aneurysm. applied to medical nanorobotics. New manufacturing 
6. Kumar S S and P S Babu. Nanotechnology. Pharma Times. 2006; 38:18-19.

7. Hellinga $\mathrm{H} \mathrm{W}$ and $\mathrm{F}$ M Richards. Construction of new ligand binding sites in proteins of known structure. I. Computer-aided modeling of sites with pre-defined geometry. J. Mol. Biol.1991; 222:763-785.http://dx.doi.org/10.1016/0022-2836(91)90510-D

8. Ummat A, Sharma G, Mavroidis C, Dubey A. Bio-nanorobotics: state of the art and future challenges. In Biomedical engineering handbook, bio-nano robotics, London, United Kingdom: Tissue engineering and artificial organs. 2005; 19:19-42.

9. Adriano Cavalcanti, Bijan Shirinzadeh, Robert A Freitas, Luiz C Kretly. Medical nanorobot architecture based on nanobioelectronics. Recent patents on nanotechnology. 2007; 1(1):1-10.http://dx.doi. org/10.2174/187221007779814745

10. Zhang J, Sabharwal C L, Tao W, Tarn T J, Xi N, and Li G. "Interactive DNA sequence and structure design for DNA nanoapplications". IEEE Transactions on Nanobioscience. 2004; 3(4):286-292. http://dx.doi.org/10.1109/TNB.2004.837918

11. Adrino Cavalacnti, et al., Nanorobot Hardware Architecture for Medical defense, Sensors. 2008; 8:2932-2958.http://dx.doi. org/10.3390/s8052932

12. Riquicha A A G et al.,Manipulation of nanoscale components with the AFM:principles and applications. IEEE. Int. Conf. Natnotechnol., Maui, HI, October 2001; 28-30:2001.

13. Sun Y, Wan K T, Roberts K P, Bischof J C, Nelson B J.Mechanical Property Characterization of the Mouse Zona Pellucida. IEEE Transaction on NanoBioScience. 2003; 2:4-8.

14. Arai $\mathrm{F}$ and Fukuda T. 3D Bio Micromanipulation. International Workshop on Microfactoryies IWMF'1998; 98:143-148.

15. Goicoechea J, Zamarreño C R, Matias I R, Arregui F J. Minimizing the photobleaching of self-assembled multilayers for sensor applications. Sens. Actuator B-Chem. 2007; 126 (1):41-47. http://dx.doi.org/10.1016/j.snb.2006.10.037

16. Das S, Gates A J, Abdu H A, Rose G S, Picconatto C A, Ellenbogen J C. Designs for ultra-tiny, special-purpose nanoelectronic circuits. IEEE Trans. Circuits Syst. I-Regul. Pap. 2007; 54 (11):2528-2540. http://dx.doi.org/10.1109/TCSI.2007.907864

17. Cavalcanti A, Shirinzadeh B, Fukuda T, Ikeda S. Hardware architecture for nanorobot application in cerebral aneurysm. IEEENano 2007 Int. Conf. Nanotechnol. 2007; 237-242

18.Leary S P, Liu C Y, Apuzzo M L I. Toward the emergence of nanoneurosurgery: Part III - Nanomedicine: targeted nanotherapy, nanosurgery, and progress toward the realization of nanoneurosurgery. Neurosurgery 2006; 58 (6):1009-1025.http://dx.doi. org/10.1227/01.NEU.0000217016.79256.16

19.Sauer C, Stanacevic M, Cauwenberghs G, Thakor N. Power Harvesting and Telemetry in CMOS for Implanted Devices. IEEE Trans Circ Sys. 2005; 52:2605-2613.http://dx.doi.org/10.1109/ TCSI.2005.858183

20. Seeman N C, DNA in a material world. Nature. 2003; 421:427-431. http://dx.doi.org/10.1038/nature01406

21.Smith S B, Cui Y, Bustamante C. Overstretching B-DNA: the elastic response of individual double-stranded and single-stranded DNA molecules. Science. 1996; 271:795-799.http://dx.doi. org/10.1126/science.271.5250.795

22. Mao C, Sun W, Seeman N C. Designed Two-Dimensional DNA Holliday Junction Arrays Visualized by Atomic Force Microscopy. Journal of American Chemical Society. 1999; 121:5437-43 http://dx.doi.org/10.1021/ja9900398

23. Pohl F M, Jovin T M. Salt-induced co-operative conformational change of a synthetic DNA: equilibrium and kinetic studies with poly (dG-dC). Journal of Molecular Biology. 1972; 67:375-96 http://dx.doi.org/10.1016/0022-2836(72)90457-3
24. Stracke R, Böhm K J, Burgold J, Schacht H, Unger E. Physical and Technical Parameters Determining the Functioning of a KinesinBased Cell-Free Motor System. Nanotechnology. 2000; 11(2):52-56. http://dx.doi.org/10.1088/0957-4484/11/2/302

25. Onion A. "RoboSnail Tackles Any Terrain - Slime Not Included", 2006. Technology and Science, ABC News, abcnews.go.com/ Technology/story?id=1525599 .

26.Cavalcanti A, Hogg T, Shirinzadeh B, Liaw H C. "Nanorobot Communication Techniques: A Comprehensive Tutorial", 2006. IEEE ICARCV Int'l Conf. on Control, Automation, Robotics and Vision, Grand Hyatt, Singapore.http://dx.doi.org/10.1109/ ICARCV.2006.345457

27. Patel G M, Patel G C, Patel R B, Patel J K, Patel M. Nanorobot: a versatile tool in nanomedicine. J. Drug Target. 2006; 14 (2): 63-67 http://dx.doi.org/10.1080/10611860600612862

28.Marchant RE, Zhang T, Qiu Y, Ruegsegger M A. Surfactants that mimic the glycocalyx. 1999. United States patent US 6759388

29. Wright E M, Sampedro A D, Hirayama BA, Koepsell H, Gorboulev V, Osswald C. Novel glucose sensor. 2005. United States patent US 0267154

30. Freitas Jr RA. Nanomedicine, Vol. I: Basic Capabilities, 1999; Landes Bioscience. http://www.nanomedicine.com/NMI.htm.

31. Ganong W F. "Review of Medical Physiology". Los Alto, 1977; CA: Lange Medical Publications, 8 th edition.

32. Chandran K B, Cardiovascular Biomechanics, New York University, 1992; 32-41.

33. Srivastava, $\mathrm{N}$ and Banerjee $\mathrm{K}$. "Performance analysis of carbon nanotube interconnects for VLSI applications", IEEE/ACM ICCAD Int'l Conf. on Computer-Aided Design. 2005; 383-390.

34. Seth S, Vincent A, Compans R W. Activation of fusion by the SER virus $\mathrm{F}$ protein: a low-pHdependent paramyxovirus entry process. $J$. Virol. 2003; 77 (11):6520-6527.http://dx.doi.org/10.1128/ JVI.77. 11.6520-6527.2003

35. Brouns S J J, Smits N, Wu H, Snijders A P L, Wright P C, Vos W $\mathrm{M}$, Oost J. Identification of a novel $\alpha$-galactosidase from the hyperthermophilic archaeon sulfolobus solfataricus. J.Bacteriol. 2006; 188 (7):2392-2399.http://dx.doi.org/10.1128/JB.188.7.23922399.2006

36. Vadali Shanthi and Sravani Musunuri. prospects for medical robots. AZojono:journal of nanotechnology online. 2007; 3:1-9.

37.Robert A. Freitas Jr. "Nanotechnology, Nanomedicine and Nanosurgery”, International Journal of Surgery 2005; 3 (12):1-4.

38. Xu W, Vijaykrishnan N, Xie Y, Irwin M J. "Design of a nanosensor Array Architecture", ACM Proceedings of the 14th ACM Great Lakes symposium on VLSI, 2004; 298-303, Boston, Massachusetts, USA.

39. Benenson Y, Gil B, Ben-Dor U, Adar R, Shapiro E. An autonomous molecular computer for logical control of gene expression. Nature. 2004; 429(6990):423-429.http://dx.doi.org/10.1038/nature02551

40. Mathieu J B, Martel S, Yahia L H, Soulez G, Beaudoin G. 2003 : MRI Systems as a Mean of Propulsion for a Microdevice in Blood Vessels. EMBC 2003.

41. Robert A. Freitas, Jr. Nanomedicine, Volume IIA: Biocompatibility, Landes Bioscience, Georgetown, TX (2005); http://www. nanomedicine.com /NMIIA.htm.

42. Cavalcanti A, Freitas Robert A Jr, Kretly Luiz C. 2004. Nanorobotics control design: a practical approach tutorial. ASME 28th Biennial Mechanisms and Robotics Conference, Salt Lake City Utah, USA.

Copyright:(c) 2011 Vijayakumar R, et al. This is an open-access article distributed under the terms of the Creative Commons Attribution License, which permits unrestricted use, distribution, and reproduction in any medium, provided the original author and source are credited. 